UCRL-JC-113366

PREPRINT

\title{
Magnetic Circular Dichroism in X-Ray Absorption and Core-Level Photoemission
}

\author{
J.G. Tobin \\ G.D. Waddill \\ T.H. Couder \\ C.A. Colmenares \\ D.P. Pappas
}

This paper was prepared for submittal to the 1993 Spring Meeting of the Materials Research Society San Francisco, CA

April 11-16, 1993

March 17, 1993

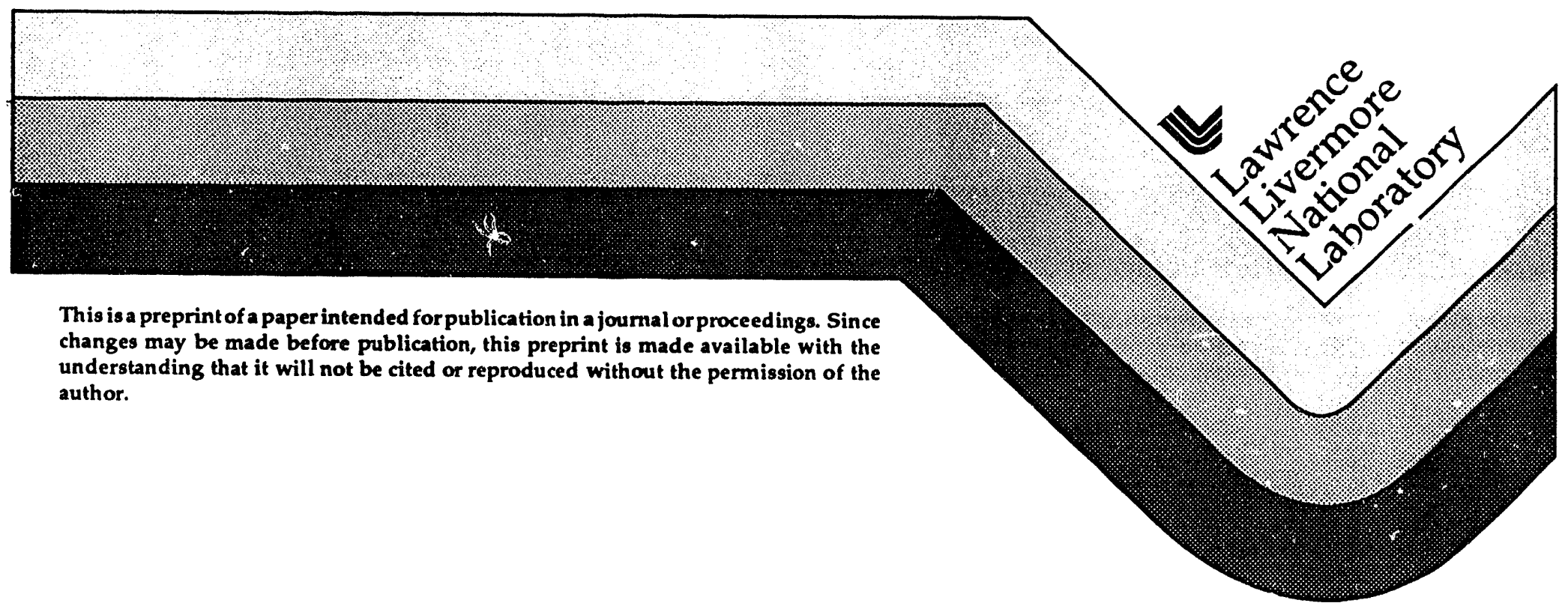

MASTER 


\section{DISCLAIMER}

This document was prepared as an account of work sponsored by an agency of the United States Government. Neither the United States Government nor the University of California nor any of their employees, makes any warranty, express or implied, or assumes any legal liability or responsibility for the accuracy, completeness, or usefulness of any information, apparat us, product, or process disclosed, or represents that its use would not infringe privately owned rights. Reference herein to any specific commercial products, process, or service by trade name, trademark, manufacturer, or otherwise, does not necessarily constitute or imply its endorsement, recommendation, or favoring by the United States Government or the University of California. The views and opinions of authors expressed herein do not necessarily state or reflect those of the United States Government or the University of California, and shall not be used for advertising or product endorsement purposes. 


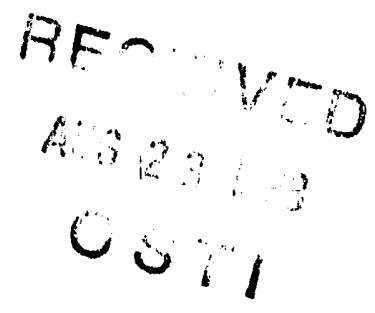

\title{
Magnetic Circular Dichroism in X-Ray Absorption and Core-Level Photoemission
}

\author{
J.G. Tobin, G.D. Waddill, T.H. Gouder, and C.A. Colmenares \\ Chemistry and Materials Science Department, University of California, Lawrence Livermore \\ National Laboratory, P.O. Box 808, Livermore, CA 94550 USA \\ D.P. PAPPAS \\ Naval Research Laboratory, Washington, DC 20375 USA
}

\begin{abstract}
Here is reported observation of magnetic circular dichroism in both $x$-ray absorption and corelevel photoemission of ultra thin magnetic films using circularly polarized $x$-rays. Iron films ( $1-4$ ML) grown on a $\mathrm{Cu}(001)$ substrate at $150 \mathrm{~K}$ and magnetized perpendicular to the surface show dramatic changes in the $L_{2,3}$ branching ratio for different $x$-ray polarizations. For linearlypolarized $x$-rays perpendicular to the magnetic axis of the sample the branching ratio was 0.75 . For films $\geq 2 \mathrm{ML}$, this ratio varied from 0.64 to 0.85 for photon spin parallel and anti-parallel, respectively, to the magnetic axis. This effect was observed either by changing the $x$-ray helicity for a fixed magnetic axis, or by reversing the magnetic axis for a fixed $x$-ray helicity. Our observation can be analyzed within a simple one-electron picture, if the raw branching ratios are normalized so that the linear value becomes statistical. Furthermore, warming the films to $\sim 300 \mathrm{~K}$ eliminated this effect, indicating a loss of magnetization in the film over a temperature range of $-30 \mathrm{~K}$. Finally, reversing the relative orientation of the photon spin and the magnetic axis from parallel to anti-parallel allowed measurement of the exchange splitting of the $\mathrm{Fe} 2 \mathrm{p}$ and $3 \mathrm{p}$ core levels which were found to be $0.3 \mathrm{eV}$ and $0.2 \mathrm{eV}$, respectively. These results are consistent with earlier studies, but the use of off-plane circularly-polarized $x$-rays from a bending magnet monochromator offers -2 orders of magnitude greater intensity than typical spin-polarization measurements. Finally, we have performed preliminary $x$-ray absorption studies of $\mathrm{UFe}_{2}$, demonstrating the feasibilty of $\mathrm{MCD}$ measurements in $5 \mathrm{f}$ as well as $3 \mathrm{~d}$ materials.
\end{abstract}

One of the basic thrusts of the investigation of nanoscale magnetic structures, whether it be ultrathin monolayer films, multilayers, or clusters, is the establishment of structure-property relationships. To be more specific, the determination of the interconnections between geometric, electronic, and magnetic structure is the fundamentally important but intermediate goal, with an eye towards the ultimate manipulation and application of this understanding. Ordinarily, efforts to measure nanoscale magnetic properties in conjunction with atomistic geometric and electronic structures runs headlong into the same problem: The magnetic perturbation tends to be a small component of the overall effect. In contrast to this, here we report giant circular dichroism in the near-edge core-level x-ray absorption of a near-monolayer metal film. An example of our data is shown in Fig. 1. The essential effect is the relative amplification of the $2 p^{1 / 2}$ peak where the magnetization and helicity are parallel, regardless of whether the magnetization is into or out of the surface plane. (Because the magnetization and $x$-ray incidence are normal to the surface, we 
call it perpendicular dichroism.) This is a direct measurement of the spin polarization and the density of the unoccupied states near $\mathrm{E}_{\mathrm{F}}$ in a ferromagnetic system.
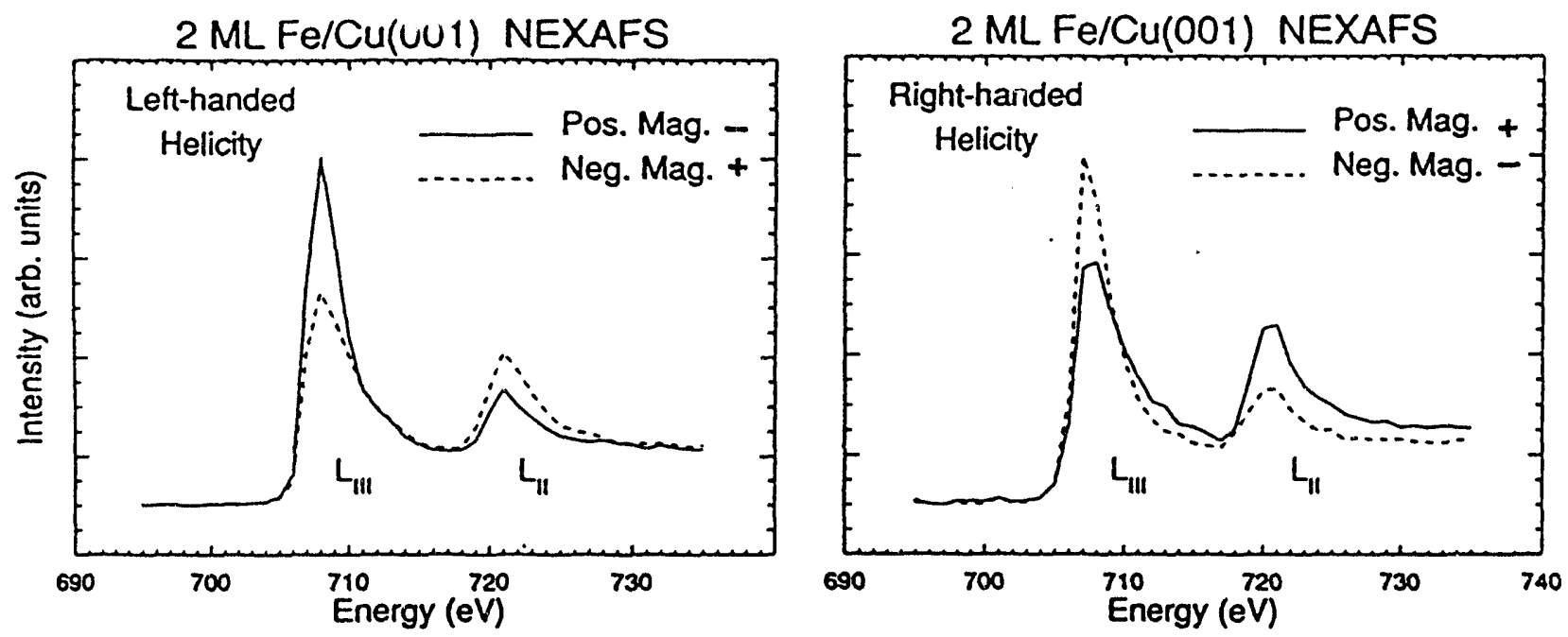

Figure 1. The near-edge $\mathrm{x}$-ray absorption fine structure (NEXAFS) dichroism of $2 \mathrm{ML}$ of $\mathrm{Fe} / \mathrm{Cu}(001)$. These are plots of absorption versus photon energy. The upper panel shows the effect of reversing the magnetization while maintaining the left-handed helicity of $x$-rays. Similarly, for the lower panel and right-handed helicity $x$-rays. Samples are perpendicularly magnetized either into (pos. mag.) or out of (neg. mag.) the surface. The symbol $+(-)$ means that helicity and magnetization are parallel (anti-parallel). The $-2 \mathrm{p} 3 / 2$ peak is at the LIII edge and the $2 \mathrm{p} 1 / 2$ peak is at the LII edge. The spectra were normalized to each other by equating the pre-edge intensity, at energies below approximately $700 \mathrm{eV}$.

The temperature dependence of the perpendicular magnetization can also be followed with this technique. Figure 2 illustrates the behavior for $4 \mathrm{ML}$ of $\mathrm{Fe} / \mathrm{Cu}(001)$. Here, we are using branching ratio (BR) as a measure of the dichroism. BR is defined in Eq. (1). I is the integrated intensity of the white line peak at each edge jump.

$$
\mathrm{BR}=\mathrm{I}\left(2 \mathrm{p}_{3 / 2}\right) /\left[\mathrm{I}\left(2 \mathrm{p}_{3 / 2}\right)+\mathrm{I}\left(2 \mathrm{p}_{1 / 2}\right)\right]
$$

We have developed a simple, one-electron picture to analyze our results, which can be summarized into the closed form analytic expression shown in Eq. (2).

$$
\mathrm{BR}=\frac{3+2(1-\alpha) \beta+2(1-\beta) \alpha}{6}=\frac{2}{3}-\frac{\left(\mathrm{P}_{\mathrm{bv}}\right)\left(\mathrm{P}_{\mathrm{c}}\right)}{6}
$$

Previously, we expressed this equation in terms of $\alpha$ nd $\beta . \alpha$ is the spin down alignment of the unoccupied $3 \mathrm{~d}$ states and $\beta$ is the degree of right circular polarization. We have now derived a more compact form, using the classical definitions of polarization. See Table I for details. 


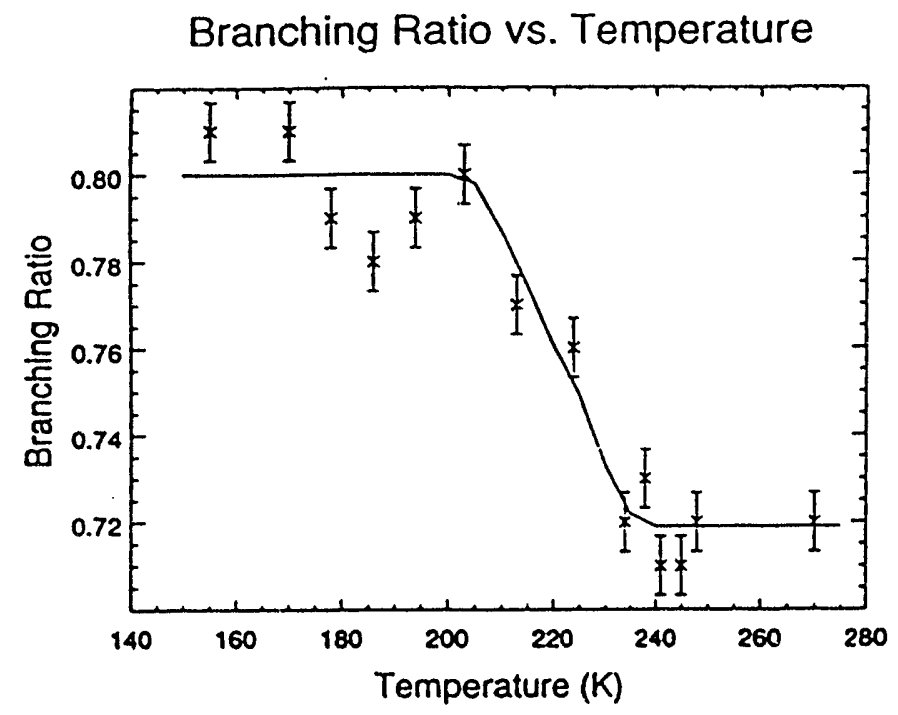

Figure 2. The temperature dependence of the magnetization of $4 \mathrm{ML} \mathrm{Fe} / \mathrm{Cu}(001)$ using NEXAFS magnetic circular dichroism as the probe. Because of the imperfect placement of the thermocouple on the sample plate, the sample temperature may be slightly higher than that shown. Error bars of approximately $\pm 1 \%$ are included for comparison but the true time error estimate is probably higher (about $4 \%$ ). A branching ratio near 0.72 corresponds to an unmagnetized sample.

\begin{tabular}{|c|c|c|c|}
\hline & Emr & Valence States & \\
\hline & Pure Down & Pure Up & Random \\
\hline$\alpha=\frac{N_{\text {UNOOC }}^{\downarrow}}{N_{\text {UNOOC }}^{\downarrow}+N_{\text {UNOCC }}^{\dagger}}$ & $1: 0$ & 0.0 & 0.5 \\
\hline 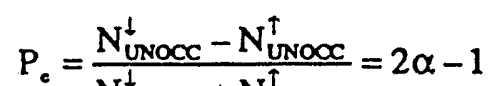 & 1.0 & -1.0 & 0.0 \\
\hline & & Photon Helicity & \\
\hline & Pure Right Circular & Pure Left Circular & Linear \\
\hline$\beta=\frac{I_{R}}{I_{R}+I_{L}}$ & 1.0 & 0.0 & 0.5 \\
\hline$P_{t v}=\frac{I_{R}-I_{L}}{I_{R}+I_{L}}=2 \beta-1$ & 1.0 & -1.0 & 0.0 \\
\hline
\end{tabular}

Table I: NUNOCC is the number of empty $d$ valence states. The symbols $\uparrow$ and $\downarrow$ denote spin up and down, respectively. $\alpha$ is the spin alignment of the $d$ valence states. $P_{e}$ is the polariza $\_$ion of the $d$ valence states. $\beta$ is the degree of right circular helicity of the $x$-rays. $P_{h v}$ is the circular polarization of the $x$-rays. $I_{R}\left(I_{L}\right)$ is the intensity of the right (left) circularly-polarized component of the $x$-ray beam. 
To use a one-electron picture, it is necessary to "normalize-out" the non-statistical manybody effects. To do this we multiply all $B R$ values by $2 / 3 / B R_{E X P}(l i n)$. This allows us to concentrate upon the effects of helicity variation. The results of our analysis, assuming $2 \mu_{\mathrm{B}} / \mathrm{Fe}$ atom ( $\alpha=25 \%$ and $75 \%)$ is shown in Table II.

Table II.

\begin{tabular}{|c|c|c|c|c|}
\hline$P$ & ersus $M$ & BREXP & BR'EXP & BRTH \\
\hline & + & 0.645 & 0.585 & 0.60 \\
\hline & - & 0.83 & 0.75 & 0.73 \\
\hline & lin & 0.74 & 0.67 & 0.67 \\
\hline$P$ & $=$ pol & & & \\
\hline $\mathbf{M}$ & $=\mathrm{ma}$ & ection & & \\
\hline $\mathrm{P}$ vs $\mathrm{M}:$ & relative & elicity and & & \\
\hline & $=$ par & & & \\
\hline - & $=$ ant & & & \\
\hline BR & $=\mathrm{bra}$ & & & \\
\hline BREXP & $=\mathrm{raw}$ & results & & \\
\hline BR'EXP & $=\exp$ & its, normali & statistical p & \\
\hline $\mathrm{BR}_{\mathrm{TH}}$ & $=$ ato & diction $2 u F$ & $25 \%, 75 \%$ & $\%$ pol. \\
\hline
\end{tabular}

We have also used MCD with core-level photoemission to measure the exchange splitting of the 2p3/2 and 2p1/2 peaks of $4 \mathrm{ML}$ of $\mathrm{Fe} / \mathrm{Cu}(001)^{2}$ as well as the Fe3p peak of $2 \mathrm{ML}$ of $\mathrm{Fe} / \mathrm{Cu}(001)$. The spectra are shown in Figures 3 and 4 . The exchange splitting of $2 \mathrm{p} 3 / 2$ peak is $0.22 \pm 0.10 \mathrm{eV}$. The apparent change in the spin-orbit splitting between the two peals is $0.33 \pm$ $0.14 \mathrm{eV}$. The exchange splitting of the $\mathrm{Fe} 3 \mathrm{p}$ is approximately $0.2 \mathrm{eV}$. There are less than those observed in the bulk (see Reference 2 and references therein). All measurements were made in remanence and with perpendicular magnetization.

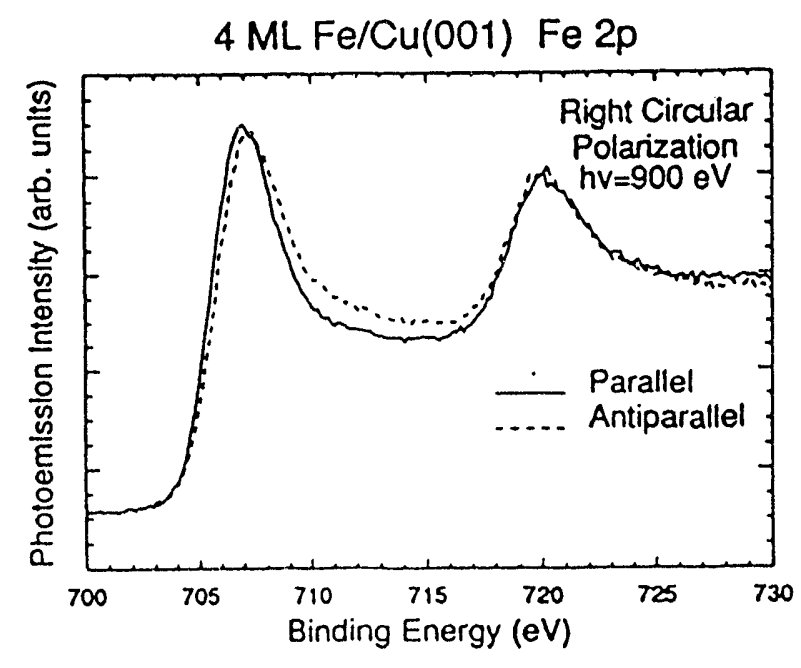

Figure $3 \mathrm{Fe} 2 \mathrm{p}$ spectra taken with $\mathrm{hv}=900 \mathrm{eV}$ and positive photon helicity. The solid lines are for parallel orientation of photon spin and sample magnetization and the broken lines for an anti-parallel orientation. The two orientations were achieved by fixing the photon helicity and reversing the magnetization of the sample. The spectra are as collected and show the differences in binding energy for the $2 \mathrm{p} 3 / 2$ and $2 \mathrm{p} 1 / 2$ levels for the two helicity and magnetization orientations. 


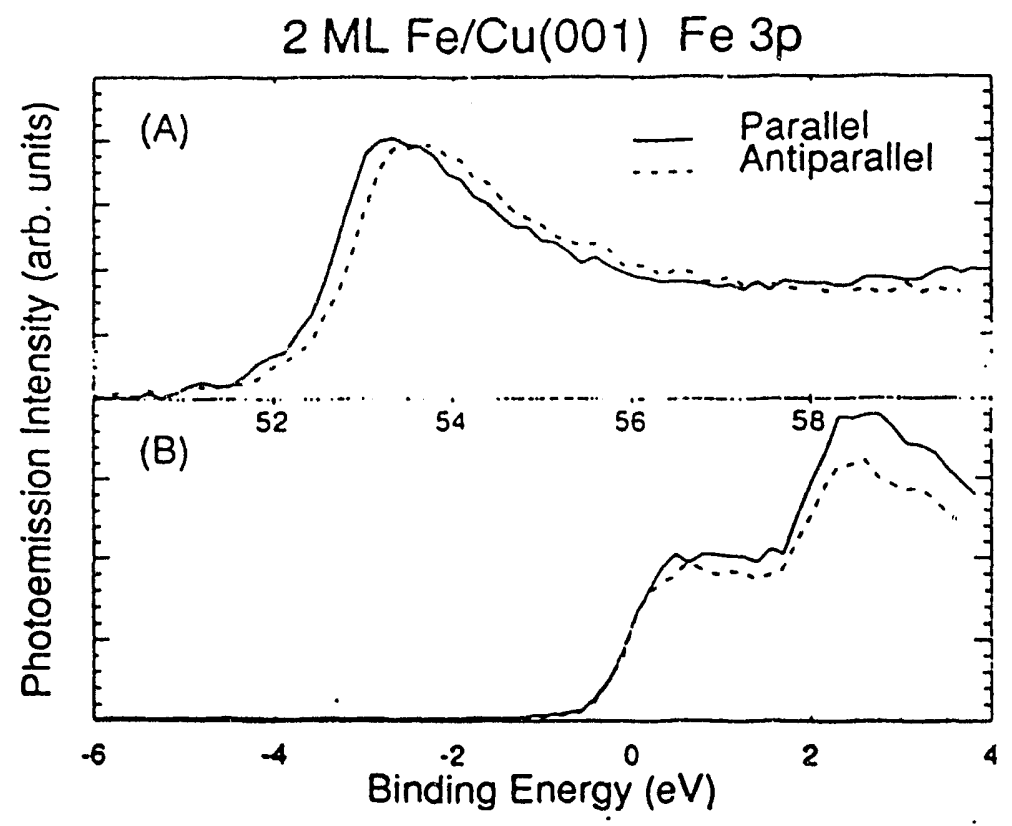

Figure 4 Magnetic circular dichroism in photoemission of the fcc-Fe 3p core level. The bottom panel shows the alignment of the Fermi edge, which allows for a direct comparison of the $3 p$ binding energies in the top panel. The sample was $2 \mathrm{ML} \mathrm{Fe} / \mathrm{Cu}(001)$, with perpendicular magnetization.

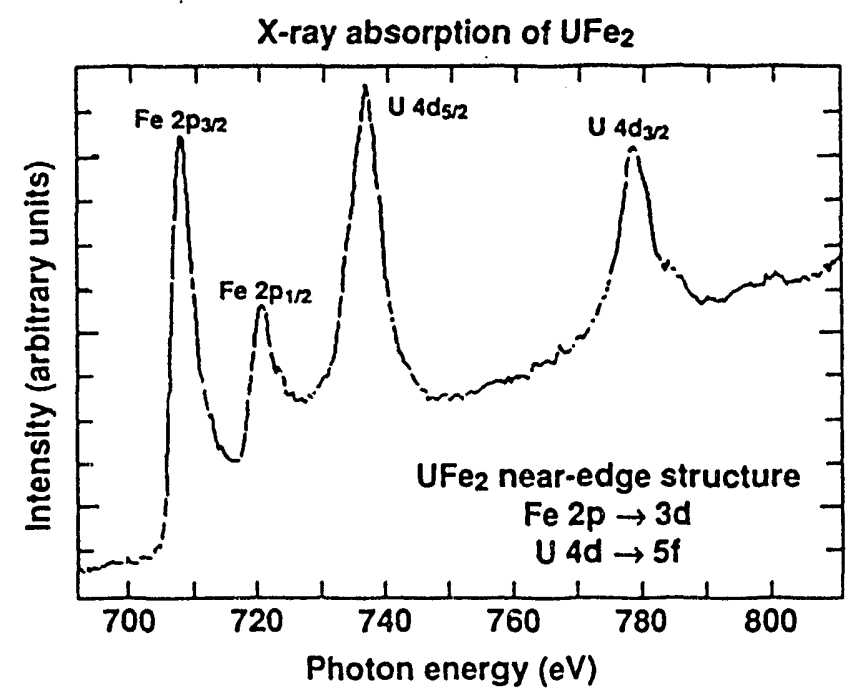

Figure 5 The near-edge $x$-ray absorption fine structure (NEXAFS) spectrum of UFe2, taken with linearly-polarized $x$-rays, is shown here. Note the strong transitions for both the Fe2p and U4d states.

Recently we began a series of experiments using near-edge-x-ray-absorption-fine structure (NEXAFS) looking for magnetic-circular-dichroism (MCD) in the magnetic actinide materials $\mathrm{UFe}_{2}$ and U-S. These and our previous experiments were performed at SSRL using the University of California/National Laboratories PRT beamline $8-2^{3}$. This SGM beamline 
permits us to access the $2 p \rightarrow 3 d$ transition of $F e\left(B^{F}=707,720 \mathrm{eV}\right)$ and the $4 d \rightarrow 5 f$ transition of $U(B F=736,778 \mathrm{eV})$. Absorption experiments with circularly-polarized $x$-rays should allow direct, elementally-specific interrogation of the magnetic and related electronic structure of these materials, in analogy with the results from magnetic $x$-ray scattering 4 . Our initial experiments utilized NEXAFS(1), but in the future they will also be expanded to include core-level photoemission ${ }^{2}$ as well.

We expect that the orders-of-magnitude improvement in brightness at the ALS will open the door for an unprecedented opportunity for both basic science using $5 f$ elements, as well as improving the overall understanding of the physical and chemical properties of uranium compound materials.

We have performed magnetic circular dichroism (MCD) experiments upon $\mathrm{Fe} / \mathrm{Cu}(001)$, using both core-level photoemission and near-edge absorption fine structure. A simple, one electron picture worked well to explain normalized branching ratio measurements. We plan to continue and expand this work, including pursuing the measurement of MCD effects in $5 f$ systems.

\section{Acknowledgments}

Work performed under the auspices of the U.S. Department of Energy by the Lawrence Livermore National Laboratory under contract No. W-7405-ENG-48. D.P. Pappas was supported by IBM Almaden when this experimental work was done. These measurements were made on Beamline 8-2 at the Stanford Synchrotron Radiation Laboratory. Beamline 8-2 is part of the UC/National Laboratories Participating Research Team (PRT) facilities ${ }^{3}$. The authors wish to thank Ms. Karen Clark for clerical support of this work.

\section{References}

1. J.G. Tobin, G.D. Waddill, and D.P. Pappas, Phys. Rev. Lett., 68, 3642 (1992) and references therein.

2. G.D. Waddill, J.G. Tobin, and D.P. Pappas, Phys. Rev. B, 46, 552 (1992).

3. L.J. Terminello, G.D. Waddill, and J.G. Tobin, Nucl. Instrum. Meth. A319, 271 (1992); J.G. Tobin, G.D. Waddill, Hua Li, and S.Y. Tong, MRS Symp. Proc. (1993), in press; K.G. Tirsell, and V.P. Karpenko, Nucl. Instrum. Meth., A291, 511 (1990).

4. D.B. McWhan, C. Vettier, E.D. Isaacs, G.E. Ice, D.P. Siddons, J.B. Hastings, C. Peters, and D. Vogt, Phys. Rev. B 느, 6007 (1990). 

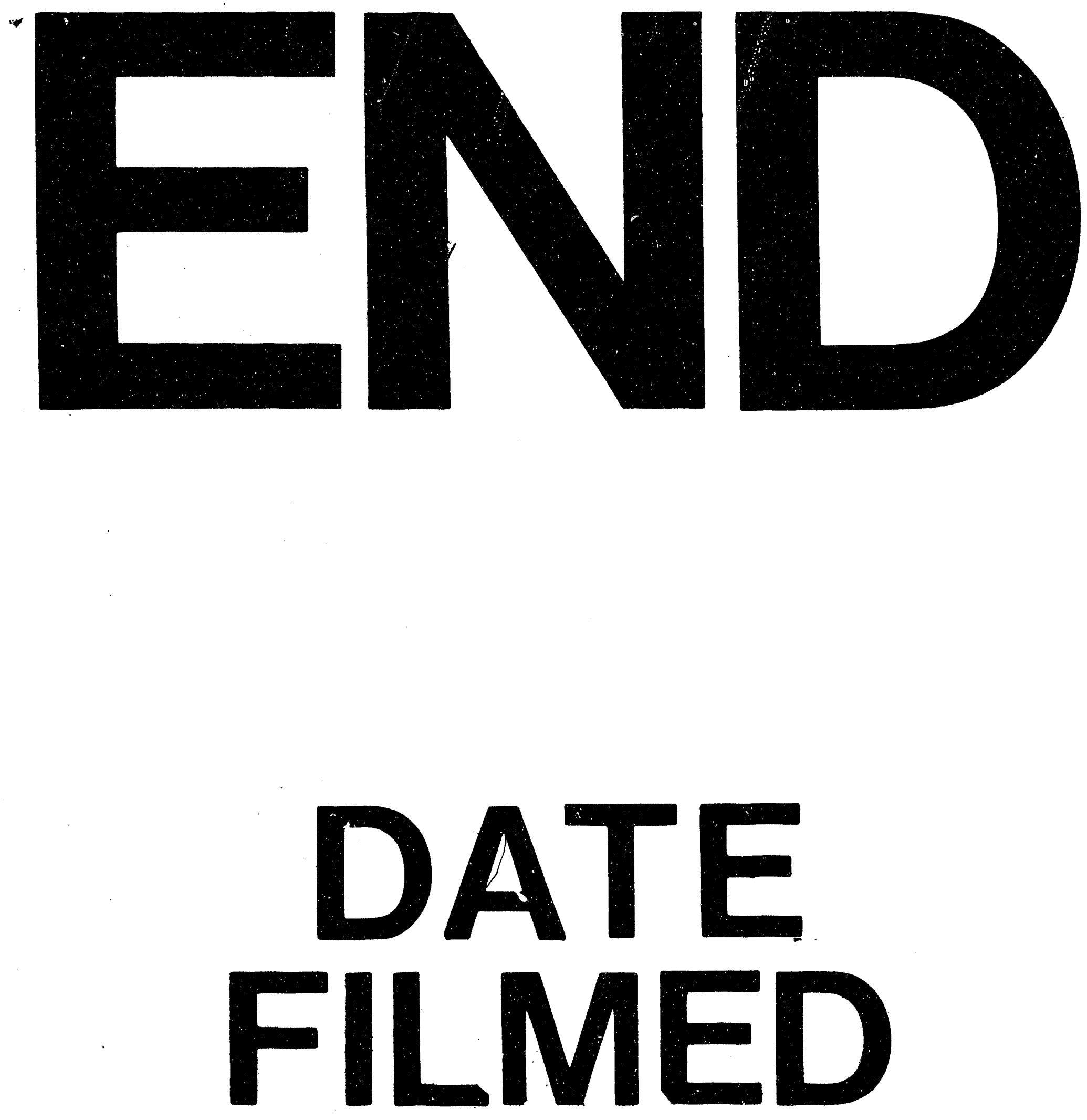

I

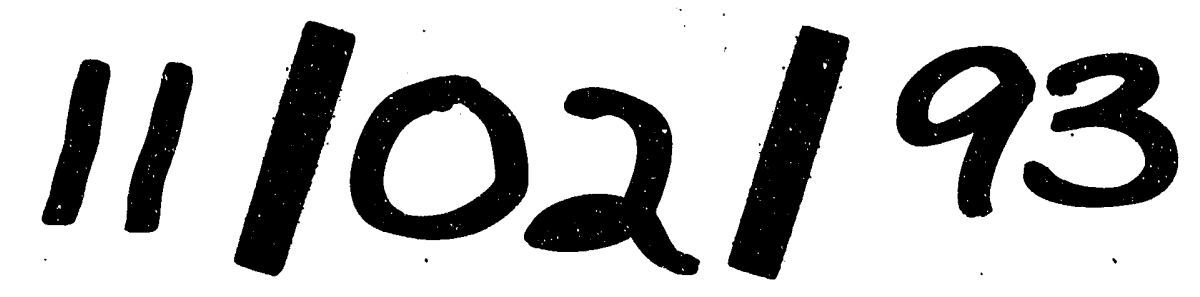


